\title{
A Multi-Robot System Based on A Hybrid Communication Approach
}

\author{
Tingkai Wang, Quan Dang \& Peiyuan Pan \\ Faculty of Computing, London Metropolitan University, London N7 8DB, UK \\ Correspondence: Tingkai Wang, Faculty of Computing, London Metropolitan University, London N7 8DB, UK. \\ Tel: 44-207-133-4383. E-mail: t.wang@londonmet.ac.uk
}

Received: March 4, 2013 Accepted: March 21, 2013 Available online: April 11, 2013

doi:10.11114/smc.v1i1.124 URL: http://dx.doi.org/10.11114/smc.v1i1.124

\begin{abstract}
Communication plays an important role in a multi-robot system. In a large system with many robots, it is difficult for all robots to exchange information at a time because of their limited communication capacities. On the other hand, sometimes there is such a situation where no explicit communication is allowed between robots. Therefore, efficient and reliable communication together with non-explicit communication is crucial for a multi-robot system. This paper presents a hybrid communication approach for a multi-robot system, which combines the explicit with implicit communications via using the prediction of robotic behaviour and a fuzzy communication approach. The hybrid communication approach contains a robot performance rule base, a fuzzy inference engine, and a semantics and grammar for communicating. Based on the hybrid communication strategy, the avoidance of collision with multiple robots in working area and multiple robots transporting a common object have been explored. The results of simulation show that the multi-robot system can complete a cooperative task successfully.
\end{abstract}

Keywords: multi-robot systems, fuzzy logic, cooperation, implicit, hybrid communication

\section{Introduction}

Recently, there has been a growing interest in multi-robot systems. Compared with a single robot, a multi-robot system increases robustness by taking advantage of inherent parallelism and redundancy (Kim, et al. 2008). Especially when doing the environmental detection, survivors searching and other complex tasks, a multi-robot system is incomparably superior. However, simply putting multiple robots together cannot constitute a multi-robot system, if they all try to function independently of each other. It may lead to a conflict, or a crash among the robots in a dynamic working environment. How to organize the multi robots with effective communication between them is a major objective of a multi-robot system. For this reason a great attention is given to multi robots communication. Various communication approaches for multi-robot systems have been developed in recent years (Aaron et al. 2011, Tarique \& Mariam, 2009 and Yan et al. 2006), which can be classified as explicit and implicit communications.

Implicit communication is usually without regard to the messages others receive. It could be based upon the environment change or perhaps the behaviour of other robots. Yan et al. (2006) applied this technique to coordinate a team of robots that have limited explicit communication. In the area of robotic search, the use of a rendezvous between two searching robots at a pre-arranged spot has been studied (Roy \& Dudek 2001). Jelle et al. (2005) applied non-communicative strategy into a multi-robot soccer team in dynamic environments. Castelfranchi et al., $(2010,2012)$ studied the theory of behavioural implicit communication. Capiluppi at al., (2013) modelled implicit communication in hierarchical Hybrid.

Although the implicit communication approach for multi robots can fulfil some tasks, explicit communication can significantly improve the flexibility and adaptiveness of a multi-robot system. Since the advent of high-performance wireless local area network (WLAN) at relatively low cost, its use for wireless communication among multi robots has become a practical proposition (Long et al. 2004).

However, for most systems with a large number of robots, communication capacity is still limited. The study on the efficient and reliable communication approaches is still considered a hot topic of research. Iqbal et al., (2009) and Kashyap \& Yan (2007) proposed dynamic message interpretation architecture for multi robots 
communication which has improved the efficiency in time and storage. Ge Ran, et al. (2007) presented an approach to improving the reliability of Wireless Sensor Networks, which uses fuzzy logic to process the information. Wang et al. (2011) presented a predict-fuzzy logic communication approach and used the approach to multi-robot system competition.

This paper, based on the paper (Wang et al. 2011), further presents a hybrid communication strategy that integrates implicit and explicit communications, and explores the strategy for multi robots cooperation. The experimental and simulation results of multi robots cooperation demonstrate that the hybrid communication strategy is reliable and efficiency, and can be used to cooperate multi robots. The remainder of the paper is organised as follows. Section 2 presents the structure of a multi-robot system. The hybrid communication approach is studied in Section 3. Section 4 explores a case study and experiments of multiple robots transporting a common object. Finally conclusions are given in Section 5.

\section{The Hybrid Approach of Explicit and Implicit Communications}

The hybrid communication system contains a robot performance rule base, a fuzzy inference engine, and a semantics and grammar for communicating etc. Those components implement both an explicit communication approach - Fuzzy Logic Communication and implicit communication - that is based on robot performance rule base, inference engine and sensor system.

\subsection{Explicit Communication Approach - Fuzzy Logic Communication (Wang et al. 2011)}

The Fuzzy Logic Communication system has following components: a fuzzy inference engine, fuzzy logic rule base, and fuzzification and defuzzification, etc. The structure of the system is shown in Figure 1. The fuzzy logic rule base is used to estimate the reliability of measurement and inference process.

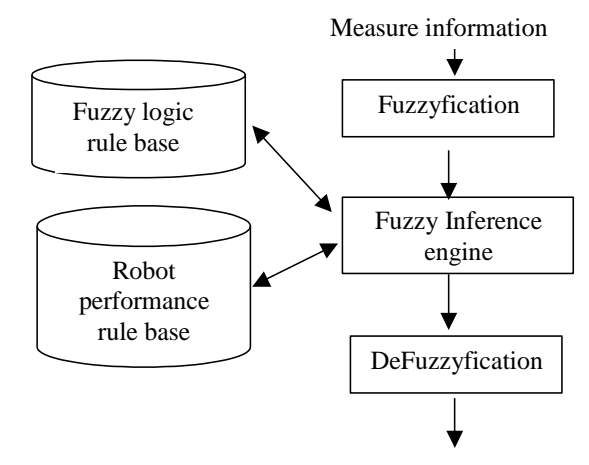

Figure 1. The Fuzzy logic communication systems

The explicit communication will follow the semantics and a grammar, which is defined as a five-element vector as follows:

$$
C \longrightarrow\left[A_{s}, A_{r}, V, O_{i}, P\right]
$$

where $A_{s}$ is an integer which represents the information sent out by a robot, $A_{r}$ represents information received by a robot, $V$ represents a verb or an instruction, $O_{i}$ represents the $i^{\text {th }}$ object (also can be one of robots), $P$ is a vector (real or fuzzy) which represents the position. For example, $\mathrm{P}=(\mathrm{X}, \mathrm{Y})$, where $\mathrm{X}, \mathrm{Y}$ can represent a real number, or a fuzzy word, such as near, medium or far. The membership functions are shown in Figure 2. The quality of position measurement is related to the distance between the sensor and an object. The closer, the more accurate (or reliable) the measurement is. Therefore, the reliability or accurate of the measurement depends on the measured distance.

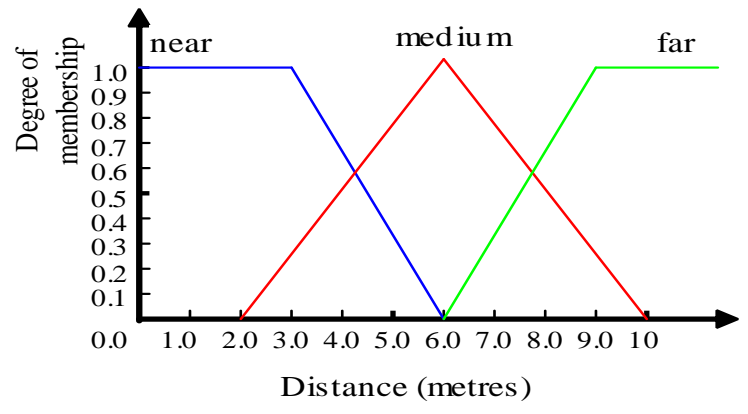


Figure 2. Fuzzification function: a distance level

Some of correspondence relationships between the number and the verb or instruction have been given in Table 1. The followings are examples for two robots (robot 01 and robot 02) communicating with each other:

Table 1. The relationship of the number with verb

\begin{tabular}{l|l|l|}
\hline & Number Verb & \multicolumn{1}{|c|}{ Verb or instruction } \\
\hline 01 & Is at \\
\hline 02 & Where is \\
\hline 03 & Speed \\
\hline 04 & Stop \\
\hline 05 & Acceleration \\
\hline 06 & Calculation \\
\hline 07 & Turn left \\
\hline$C_{1} \longrightarrow[02,01,02,03,(0,0)]$ & Turn right \\
\hline$C_{2} \longrightarrow[01,02,01,03,(10$, far $)]$ & ..... \\
$C_{3} \longrightarrow[01,02,03,03,(0.07,0.07)]$ & \\
\hline 08 &
\end{tabular}

$C_{l}$ represents that robot 02 asks robot 01 where is the position of object $03, C_{2}$ and $C_{3}$ mean that robot 01 is replaying robot 02 : the position of object 03 is at the $\mathrm{X}$-coordinate of 10 and Y-coordinate of far. And the speed of object 03 is $0.07 \mathrm{~m} / \mathrm{s}$ in both $\mathrm{X}$ and $\mathrm{Y}$ directions.

\subsection{Implicit Communication}

The robot performance rule base contains the robot codes of conduct, which robots should follow. Based on the information received from the measurement (sensor) system, the inference engine can predict behaviour of robots therefore achieve the implicit communication.

The performance rules base contains the robot traffic rules, such as keeping on the left side of road, speed limits, and passing through cross roads, etc. Surely, different robots can have a different code of conduct.

Typical rules for negotiating the crossroads are as follows:

- If robot 1 is at the fork of a crossroad and it will cross the route required by robot 2 , then robot 2 will wait for robot 1 to pass.

- If multiple robots arrive at a crossroad at the same time, the one at right side will pass through the crossroad first.

- If a static obstacle in the path then the robot needs to start the new path planning calculation.

- If a moving obstacle in the path the robot needs to calculate the waiting time.

- $\quad$ And so on.

Based on such performance rules, robots can predict each other's behaviour (or implicit communication) and have tacit cooperation. The implicit communication is based on a sequence of reasoning. Therefore the inference engine, performance rules base and measurement information are the three key elements.

The implicit communication can sort out some of communication problems for a multi-robot system particularly when it is difficult to exchange information at the time or the information is kept secret. However the implicit communication is not clear and fast enough sometimes. The explicit communication is still a commonly used communication approach. Therefore combining the explicit communication with implicit communication, while taking the advantages and avoiding the disadvantages of them, is an efficient and reliable approach. In our multi-robot system, the hybrid strategy is: If one method is not working, another is deployed; the implicit communication will be used mostly to predict the behaviour of other robots, and the explicit communication will be used mostly to give instructions. 
Based on the hybrid communication methods, multiple robots can cooperate to complete a complex task. That is, the superiority of a multi-robot system over a single robot can be achieved. However, the design of a multi-robot system, the form of cooperation and the requirements vary with different purposes. It is difficult to find a uniform cooperation algorithm for all situations.

\section{Communications Protocol}

The communication protocol for explicit communication is important for a mobile robots system. In our system, it consists of two levels: the symbol transport and the message content. The symbol transport is basic for symbols transform. It provides a channel between a sender and a receiver over which symbols are exchanged. The content level provides formats of the symbols that can be understood between a sender and a receiver therefore achieve the purpose of communication. The structure of the communication protocol is shown in Figure 3

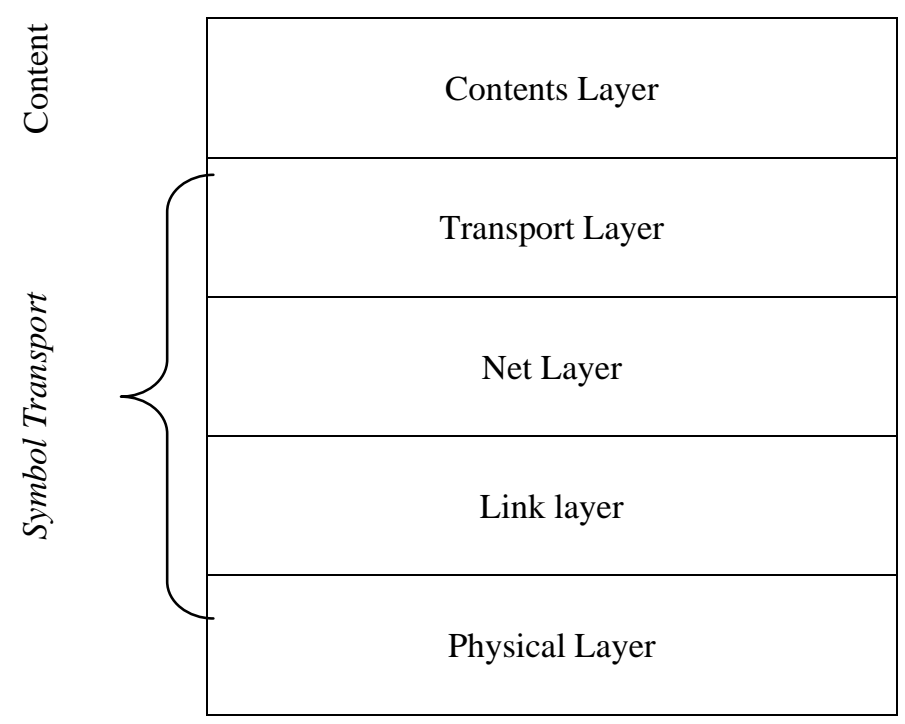

Figure 3. Structure of the communication protocol

\subsection{Symbol Transport}

The symbol transport $t$ is completely independent of the information represented at the content level. Thus, the information transmission aspect of robot communication can be treated as an instance of the more general situation of inter-computer communication. The symbol transport consists of four layers, Physical layer, Link layer, Net layer and Transport layer, which are borrowed from the OSI model.

\section{Physical layer:}

The physical layer consists of the basic networking hardware transmission technologies of a network. It is a fundamental layer underlying the logical data structures of the higher-level functions in a network. The physical layer defines the means of transmitting raw bits rather than logical data packets over a physical link connecting network nodes. The bit stream will be grouped into code words or symbols and converted to a physical signal that is transmitted over a hardware transmission medium.

\section{Link layer:}

The link layer is the lowest layer in the Internet Protocol Suite, the networking architecture. It is the group of methods or protocols that only operate on a host's link. The service on this layer providing to the net layer is a transmission channel between neighbouring robots. Its functions include sending and receiving data to and from neighbouring robots.

\section{Net layer:}

The net layer will provide a routing of packets between any pair of robots for the transport layer. To enable routing of packets, one very fundamental issue is of addressing; each robot in the system should be uniquely identified. IP addresses are used in the network. Each IP address is of 32 bits. In our robots system, the packet length is very small, and 8 bits are used for addressing.

Transport layer: 
Transport layer contained the TCP/IP model that is the foundation of the Open Systems Interconnection (OSI) model of general networking. This layer provides end-to-end communication services for applications. In a robot, there might be multiple applications running simultaneously, for example, the robot might be detecting at the same time when it is moving. To establish a communication flow between two applications, transport layer offers the abstraction of ports.

\subsection{Content Level}

At the content level contents layer should have the ability to convert the received symbols to information which robot could understand. The received symbol consists of a sequence of five-element vectors, each of the five-element vector is as follows:

$$
C \longrightarrow\left[A_{s}, A_{r}, V, O_{i}, P\right]
$$

The database of the relationship of the number with verb (refer to Table 1) and related process functions are presented in this layer.

\section{Application Case Study and Experiments -Multiple Robots Transport a Common Object}

We now apply the hybrid approach of explicit and implicit communications to a situation of multi robots cooperation. The simplest example is two robots transporting a common object in a multi-robots working environment. It is required that these two robots should keep the same spacing within a predetermined range throughout the whole process of motion and avoid conflict with other robots or obstacles. According the robot's kinematic model (Wang, et al. 1996), this problem can be described in theory for $\operatorname{robot}_{\mathbf{i}}(\mathrm{i}=1,2)$ as follows:

$$
\begin{gathered}
x_{i}(t)=x_{i}(0)+\int_{0}^{t} V_{i}(t) \cos \theta_{i}(t) d t \\
y_{i}(t)=y_{i}(0)+\int_{0}^{t} V_{i}(t) \sin \theta_{i}(t) d t \\
\theta_{i}(t)=\theta_{i}(0)+\int_{0}^{t} \frac{V_{i}(t) \tan \varphi_{i}(t)}{L_{i}} d t \\
D^{*}=\sqrt{\left(x_{i}(t)-x_{j}(t)\right)^{2}+\left(y_{i}(t)-y_{j}(t)\right)^{2}}, \quad(i \neq j, i, j=1,2)
\end{gathered}
$$

or:

$$
\left|D^{*}-\sqrt{\left(x_{i}(t)-x_{j}(t)\right)^{2}+\left(y_{i}(t)-y_{j}(t)\right)^{2}}\right| \leq \varepsilon, \quad(i \neq j, i, j=1,2)
$$

where $x_{i}(0)$ and $\mathrm{y}_{i}(0)$ are the initial stage (primary) coordinates of the robot, $\varphi_{i}$ is the steering angle, $V_{i}(t)$ is the speed, $\theta_{i}(t)$ is the angle between the robot and the x axis, $L_{i}$ is the length, $\mathrm{D}^{*}$ is the distance between the supports of two robots (which should be kept constant), and $\varepsilon$ is the allowable range of the displacement between the two robots. Usually, $L_{o}>D^{*}$, where $L_{o}$ is the length of the common obstacle.

Equations (1)-(3) combine the kinematics model of the robots, and equation (4) or (4') is a constraint (restrictive) condition for two robots' motion. This kind of task can only be completed by cooperative robots.

There are two forms for transportation of a common object: serial (i.e., one robot in front of another) and parallel (i.e., two robots run in line abreast). If there is no obstacle in the working space, these forms of transportation are acceptable. However, in order to avoid an obstacle, the serial transport form is preferable. In this paper we will only consider the form of serial as shown in Figure 4. 


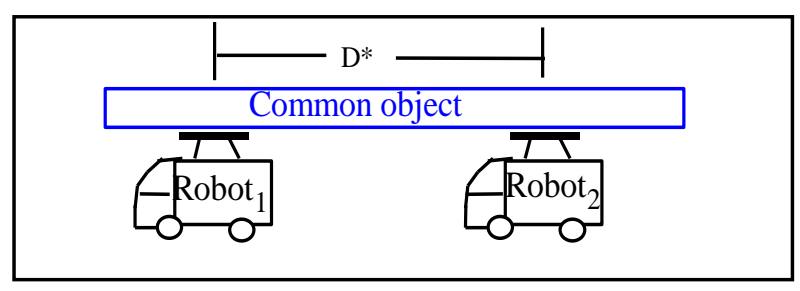

Figure 4. Two serial robots transport a common object

From equations (1)-(4), the requirement for two robots to transport a common object is to maintain the same speed and heading. According to our multi-robot system structure design, this can be achieved because of the good communication ability. The leading robot (robot 1 in Figure 4) needs to avoid a collision with other robots in the same working environment based on the robot's performance rules or implicit communication approach and sends information on its speed $V(t)$ and steering angle $\varphi(t)$ to the rear robot (robot 2 in Figure 4). On receiving any information (instructions) from robot 1 , robot 2 controls its speed and direction by its own controller according to the instructions and the desired motion (path planning). Figure 5 shows the structure of the required control system, which is based on robots' communication.

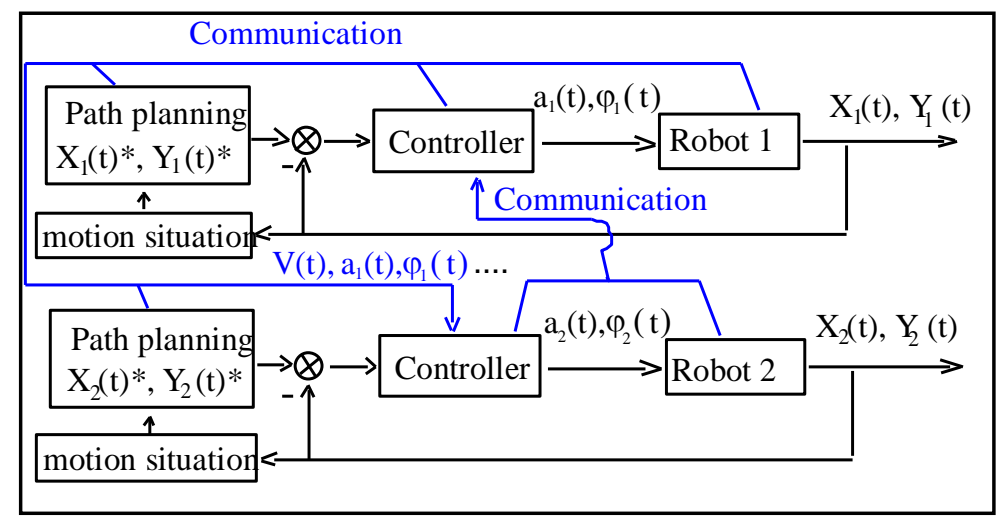

Figure 5. Structure of control system

Path planning is somewhat different from that of a single robot system. In a cooperating system, the path planning needs to consider the motion of another robot in the same working environment, in addition to the expected locus of the moving object. Therefore the path planning approach should be event-based and need the hybrid communication approach to support. The basic idea of the event-based planning and control theory is to introduce a new motion reference variable that is changing with time, but directly related to the sensory measurement of the system, and the performance of move obstacle can then be predicted. Instead of time, the desired system output is parameterized by the new motion reference variable. The latter is designed to efficiently carry the sensory information needed for the planner to adjust or modify the original plan to form a desired output (Wang et al. 2010, 1999). As a result, for any given time instant, the action plan is a function of the system output. This creates a mechanism to adjust and modify the plan based on the sensory measurement. The path planning of two robots transporting a common object can be described as follows. Suppose that the expected locus of the object is $E_{o}(x, y)$, the loci of robot 1 and robot 2 are $E_{l}(x, y)$ and $E_{2}(x, y)$, then the path planning can be described as follows:

$$
\begin{aligned}
& E_{1}(x, y)=f_{1}^{*}\left(E_{o}, D, E_{2}\right) \\
& E_{2}(x, y)=f_{2}^{*}\left(E_{o}, D, E_{1}\right)
\end{aligned}
$$

where the map $f_{l}^{*}$ satisfies the following relationship: The distance between $E_{l}(x, y)$ and $E_{2}(x, y)$ is $\mathrm{D}$, and the distance between $E_{l}(x, y)$ and $E_{o}(x, y)$ is less than D, that is, 


$$
\begin{aligned}
& \left\|E_{1}(x, y)-E_{2}(x, y)\right\|=D \\
& \left\|E_{1}(x, y)-E_{o}\right\|<D
\end{aligned}
$$

Similarly, the map $f_{2}{ }^{*}$ satisfies the relationship as follows:

$$
\begin{aligned}
& \left\|E_{1}(x, y)-E_{2}(x, y)\right\|=D \\
& \left\|E_{2}(x, y)-E_{o}\right\|<D
\end{aligned}
$$

These equations have multiple solutions. For simplicity, equation (5) and equation (5') can be replaced by the following:

$$
E_{1}(x, y)=f_{1}^{*}\left(E_{o}, D\right)
$$

That is,

$$
\left\|E_{1}(x, y)-E_{o}\right\|<D
$$

Thus equations (1)-(6) give a mathematical description on the trajectory planning problem where two robots transport a common object.

The significance of equation (5") is that only one of two robots needs to consider the constraint condition $\left\|E_{l}(x, y)-E_{o}\right\|<D$, when it is planning a path. Therefore it can still use the single robot path planning approach (Wang et al. 2010). The other robot's path planning should be based on distance D. D can be chosen as a new motion reference variable or motion situation. In fact, the path planning and control of robot 1 (leading robot) uses a fuzzy algorithm, which can ensure a suitable path and control result. Robot 2 (auxiliary robot) can also use the fuzzy algorithm, but it should pay more attention to the motion situation and hence the rule control is different from that of robot 1 . Some of the rules are:

If $V_{1}(t)=V_{2}(t)$ and $|D-D *| \leq \varepsilon / 2$, then maintain speed.

If $V_{1}(t)-V_{2}(t) \geq 0$ and $\mathrm{D}>\mathrm{D}^{*}$, then speed up.

If $V_{1}(t)-V_{2}(t) \geq 0$ and $\mathrm{D}<\mathrm{D}^{*}$, then maintain speed.

If $V_{1}(t)-V_{2}(t) \leq 0$ and $\mathrm{D}>\mathrm{D}^{*}$, then maintain speed.

If $V_{1}(t)-V_{2}(t) \leq 0$ and $\mathrm{D}<\mathrm{D}^{*}$, then reduce speed.

$$
\text { ...... }
$$

where $D=\sqrt{\left(x_{1}(t)-x_{2}(t)\right)^{2}+\left(y_{1}(t)-y_{2}(t)\right)^{2}}, \quad V_{1}(t)$ and $V_{2}(2)$ are the velocities of robot 1 and robot 2 .

The steering control rules for robot 2 are as follows:

$$
\begin{aligned}
& \text { If } \varphi_{1}=0 \text { and } \varphi_{11}=0 \text {, then } \varphi_{2}=0 ; \\
& \text { If } \varphi_{1} \neq 0 \text { and } \varphi_{11}=0 \text {, then } \varphi_{2}=\varphi_{1} \text { after T seconds; } \\
& \text { If } \varphi_{1} \neq 0 \text { and } \varphi_{11} \neq 0 \text {, then } \varphi_{2}=\varphi_{1} ; \\
& \text { If } \varphi_{1}=0 \text { and } \varphi_{11} \neq 0 \text {, then } \varphi_{2}=0 \text { after T seconds; }
\end{aligned}
$$

Where $\varphi_{1}$ and $\varphi_{2}$ are the steering angles of robot 1 and robot $2, \varphi_{11}$ is $\varphi_{1}$ before sampling, and $T=\frac{D^{*}}{V(t)}$.

A similar approach can be applied to transport a common object by a group of $(n+1)$ robots in cooperation. One robot (leader) sends all information about its planned speed $V(t)$ and steering angle $\varphi(t)$ to the others according to the desired motion (path planning). Its path planning control can be based on the single robot's path planning approach. The other $\mathrm{n}$ robots will control their speed and direction respectively by controllers according to the desired motion (path planning) and the instructions. The instructions and the distances between other robots are the new motion reference variables for $\mathrm{n}$ auxiliary robots for which path planning and control is event-based. 


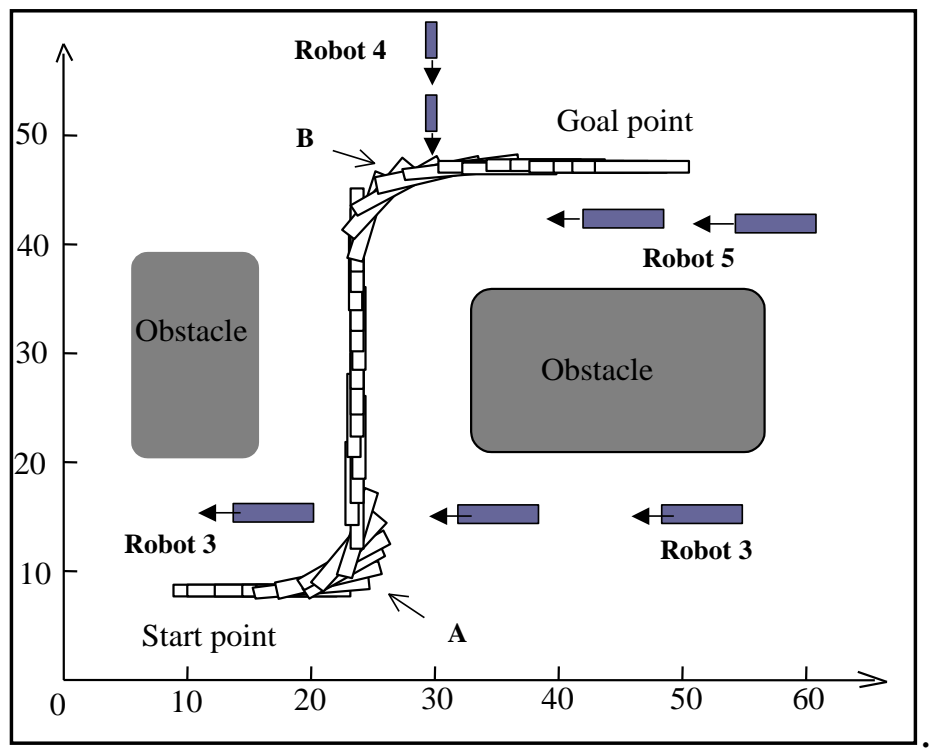

Figure 6a. Simulation result of two robots transporting a common object in multi-robotics working environment

Simulation: Suppose that the length of each robot is $L_{l}$ (or $\left.L_{2}\right)=1$ meter, the loading capacity is 500 kilograms, the length of object is $L_{o}=5$ meters, and its weight is 700 kilograms. The range of the object's allowable deviation between the two robots is $\varepsilon=0.5$ (meter). The distance between the supports of the two robots $D^{*}$ is 4 meters. Figure 6a shows the simulation result and Figure 6b shows the distant error curve deviation between the two robots. From the Figure 6b, it is clear that the error is largest when the robots turn a corner. However the error never reaches more than 0.4 meter in the control process. Clearly, this cooperation method works successfully.

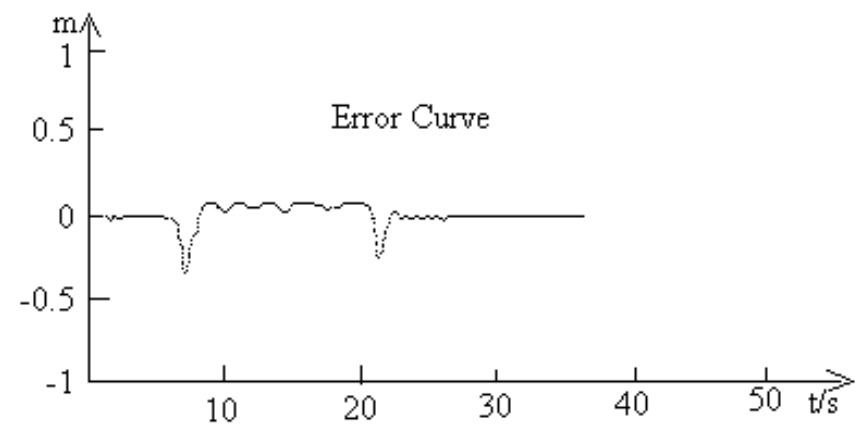

Figure $6 \mathrm{~b}$. The error curve (deviation between robots)

How to avoid a conflict with other robots in the multi-robots working environment is a key for the whole system to succeed.

In the simulation process, the implicit communication, or robot traffic rules, has sorted out the problem successfully: robot 4 waited at point B (in Figure 6a) when robot 1 and 2 have indicated that they are going to turn to right. At point A (in Figure 6a), robots 1 and 2 need to wait robot 3 to pass, then they will continue moving. If there is a rule: "the light-loaded robot should avoid heavy-loaded robot" in the working environment, then robot 3 will wait at point A until robots 1 and 2 have passed. The robot traffic rules can have different contents in different working environments.

\section{Conclusions}

A multi-robot system has been widely used in auto-warehouse and large working place. However achieving the cooperation in a multi-robots team is a very complex task. It covers a wide range of disciplines and technologies, including distributed artificial intelligence, computer communication, control system, cooperation and 
competition strategy, etc. Some solutions to certain types of problems are even unknown to humans. Because of its diversity, it is hard to make a comparison of different approaches due to lack of commonly accepted test standards and procedures. The research platforms used differ greatly, as do the key assumptions used in different approaches.

This paper presented the hybrid communication strategy of combining the explicit with implicit communications for multi-robot system. A five-element vector is used to describe the semantics and grammar for robot communication. Robot performance rule base (or traffic rules) is introduced into the system that can predict behaviour of robots and then achieve the implicit communication. It significantly reduces overheads for explicit communication amongst robots. Based on the hybrid communication strategy, avoiding a collision among multiple robots in a working area and multiple robots transporting a common object have been explored in a multi robots working environment. The results of simulation show that the multi-robots can cooperate successfully to complete tasks based on the hybrid communication approach presented.

\section{References}

Aaron B. St. Clair, Amin Atrash, Ross Mead, \& Maja J. Matarić. (2011). Speech, Gesture, and Space: Investigating Explicit and Implicit Communication in Multi-Human Multi-Robot Collaborations, Refereed Workshop 2011 American Association of Artificial Intelligence Spring Symposium on Multi-Robot Systems and Physical Data Structures (SS-11-09).

Castelfranchi, C., et al., (2010). Behavioral Implicit Communication (BIC): Communicating with Smart Environments. International Journal of Ambient Computing and Intelligence (IJACI), 2(1), 1-12. http://dx.doi.org/10.4018/jaci.2010010101

Castelfranchi, C., et al., (2012). Behavioral Implicit Communication (BIC): Communicating with Smart Environments via our Practical Behavior and Its Traces. Innovative Applications of Ambient Intelligence: Advances in Smart Systems, 1-12.

Capiluppi M., \& Segala, R. (2013). World Automata: a compositional approach to model implicit communication in hierarchical Hybrid, 3rd Workshop on Hybrid Autonomous Systems 2013, 17 March 2013, Rome, Italy.

Long, X. L., Jiang, J. P., \& Xiang, K. (2004). Towards Multi-robot Communication, proceeding of IEEE International Conference of Robotics and Biomimetics, ROBIO, 307-312.

Iqbal, J., Yousaf, M. M., \& Awais, M. M. (2009). A scalable approach of message interpretation by demonstrations for multi-robot communication. Proceeding of IEEE 13th International Multitopic Conference, INMIC 2009. pp1-6.

Jelle, R. K., Matthijs, T. J., \& Spaanand, N. V. (2005). Non-communicative multi-robot coordination in dynamic environments. Robotics and Autonomous Systems, 50(2-3), 99-114.

Kashyap, S., \& Yan, M. (2007). Communication-Efficient Dynamic Task Scheduling for Heterogeneous Multi-Robot Systems. Proceedings of the 2007 IEEE International Symposium on Computational Intelligence in Robotics and Automation, pp230-235, USA, June 20-23.

Kim, S. C., Shin, K. H., Woo, C. W., Eom, Y. S., \& Lee, J. M. (2008). Performance Analysis of Entropy-based Multi-Robot Cooperative Systems in a MANET. International Journal of Control, Automation, and Systems, 6(5), 722-730.

Ran, G., Zhang, H., \& Gong, S. (2010). Improving on LEACH Protocol of Wireless Sensor Networks Using Fuzzy Logic. Journal of Information \& Computational Science, 7(3), 767-775.

Roy, N., \& Dudek, G. (2001). Collaborative robot exploration and rendezvous: algorithm, performance bounds and observations. J. Autonomous Robot, 11(2), 117-136. http://dx.doi.org/10.1023/A:1011219024159

Tarique, H., \& Mariam, Y. (2009). A Fuzzy Approach to Energy Optimized Routing for Wireless Sensor Networks. The International Arab Journal of Information Technology, 6(2).

Wang, T., Mehdi, Q., \& Gough, N. (1996). Kinematics models of autonomous guided vehicles and their applications. Proc. of ISCA 5th International Conference, Reno Nevada, USA. 207-211.

Wang, T., Mehdi, Q. H., \& Gough, N. E. (1999). An integrated navigation system for AGVs based on an environment database. International Journal of Computers and Their Applications, 6(1), 14-24.

Wang, T., Dang, Q., \& Pan, P. (2010). A Path Planning Approach in an Unknown Environment. International Journal of Automation and Computing (IJAC), 7(2), 310-316. http://dx.doi.org/10.1007/s11633-010-0508-6 
Wang, T., Dang, Q., \& Pan, P. (2011). A Predict-Fuzzy Logic Communication Approach for Multi Robotic Cooperation and Competition. Journal of Communications, 6(3), 225-231.

Yan, M. J., Nickerson, V., \& Jing, G. (2006). Multi-robot Aggregation Strategies with Limited Communication. Proceedings of the IEEE/RSJ International Conference on Intelligent Robots and Systems, Beijing, China.

\section{(c) EY}

This work is licensed under a Creative Commons Attribution 3.0 License. 